\title{
The Connection Between the Effectiveness of the N95 masks, and the Process of Recharging Them
}

\author{
Joshua Li ${ }^{1}$ and Danny Zhang ${ }^{1}$ \\ ${ }^{1}$ Exploring Scholar, North York, ON, CA
}

\section{ABSTRACT}

Before reading this paper, many people might have thought that N95 masks are like a very secure and efficient strainer, where the gaps are so small that the particles can't pass through. However, N95 masks don't work like that. N95 masks have 2 defense systems. One which uses gravitational force to trap particles by touching them, and the other which uses a static electric field to attract particles of all sizes. This is done by using polypropylene fiber, which has both a positive and negative charge. However, the charge on the polypropylene will slowly regrade when being used, with a maximum usage length of one day. This leaves many hospitals with an insufficient amount of N95 masks, but it is possible to reuse N95 masks by decontaminating them to remove any possible risk of COVID-19 on the mask, then recharging the polypropylene so it regains its filtering effect again. This will allow the mask to be used more than once, so hospitals will not run out of masks, and there will be more N95 masks for the public if they are not in high demand anymore. This paper will aim to explain the physics of filtration and care/hygiene of N95 masks, as well as the physics of recharging N95 masks, which is important to helping you protect your own health and avoid the risk of getting COVID-19.

\section{Introduction}

During the pandemic, there has been confusion and worry on how N95 masks work, and if wearing masks truly helps protect you from COVID-19. There are currently many types of masks, from cloth masks to N95s. Each type of mask has its own uses and different filtering efficiencies, but many people do not understand what makes the masks different and why some work better than others.

There are many steps to properly protecting yourself with a N95 mask. This will be broken down and explained through the physics behind N95s, the material used in N95s, the difference between N95 masks and surgical masks, and how to decontaminate, recharge, and reuse N95 masks.

\section{Context}

N95 masks are one of the highest demanding masks during the COVID-19 pandemic due to its high efficiency of blocking out the virus. The name N95 defines the mask's standards clearly. The N is a Respirator Rating Letter Class. It stands for "nonoil", so it is safe to use if there are not oil related particles around. The 95 in the name means that the mask has a $95 \%$ efficiency of blocking out the virus.

The N95 mask was originally made in the 1990s by a scientist named Peter Tsai [T.H.I U.S. Embassy Tbilisi (2020)]. He led a research team and developed air filters, and was later made into a mask, using a material called polypropylene fiber, which has the properties to create an electric field that attracts particles of all sizes. They tested with dust and other small particles, and $95 \%$ of the particles was trapped by the polarization before it passed through the mask. The mask was then used by construction workers to block out dust and other micro particles. In 1996, the Center for Disease Control discovered that the N95 can also be used to block out viruses and bacteria, and after 
combining it with the design of a surgical mask made by $3 \mathrm{M}$, it was introduced as a standard mask worn by medical professionals around the world.

One common misunderstanding of the N95 masks is that they are like a type of filter, and the gaps between filters are very small, so most of the particles that pass through are blocked by it. However, this is quite different from how N95 masks work. The goal of the N95 masks is simply for the particles to touch the fibers. Once they do, the particles become stuck to the fibers. This is not because the fibers are special, it is because at the microscopic level, everything sticks because the weakly attracted force between molecules are more than enough to keep the molecules stuck to the fibers. Instead of a shutter to keep out flies, a N95 is more like a spider web that sticks to insects of all sizes, as long as they touch the web.

There are 2 main ways a N95 mask can make the particles touch the fibers. The first way is through have many fibers stacked up in front and behind each other. This increases the chance of a particle touching a fiber by a tenfold and can trap particles of all sizes. Particles that are smaller than 0.1 micrometer are so light that they travel in a zig zag pattern, which makes it easy to touch a fiber. Particles that are bigger than 1 micrometer travel in a straight line due to their inertia. However, particles around 0.3 micrometers travel with the flows with the air current, thus making it more likely for them to avoid the fibers. However, the N95 has another line of defense against the particles. As mentioned before, the polypropylene in N95 masks creates an electrical field that can cause even neutral particles to develop an internal electrical imbalance. The fibers are like permanent static electricity, and just like how you can magnetise a piece of iron, you can also electrify a piece of plastic to give it an electric charge. They end up gaining a strong and long-lasting ability to attract particles and are able to attract 10 times as many particles as normal fibers.

\section{Observations and Experiments}

\section{How does the polypropylene in the N95 work?}

Polypropylene fiber is a synthetic fiber that was transformed from $85 \%$ propylene and can be used in a variety of applications. Polypropylene can be processed into wrapping film for packaging or made into fiber for carpet and clothing. Polypropylene has a few properties that makes it the second most widely produced commodity plastic. It has excellent chemical resistance, can be translucent or opaque, and is also lightweight.

Normally clothes and furniture have a woven pattern, where everything is patterned. However, nonwoven materials have a random arrangement of fibers, causing it better filter particles while remaining highly breathable. Two common types of polypropylene that was used in masks were Spunbond and Meltblown [C. Clase, C.D. Lannoy, S. Laengert, (2020)] There were a few published data about the filtration efficiency of Spunbond polypropylene, but one layer with a rating of 68 grams per square metre was recommended, or 2 layers of a less dense one. Meltblown polypropylene is more commonly used in the middle layer of many certified medical masks, such as N95s. It filters very well, but in exchange there is a low supply of it in Canada. It also cannot be washed; thus it was not recommended to be used in reusable nonmedical masks. These filters are sold commercially and may contain Spunbond, Meltdown, or many other materials, and although there are no standards on them in Canada, they were all designed to be used once.

\section{N95 masks vs Surgical masks}

N95 masks and surgical masks may look similar, but they are in fact very different and they were intended for completely different purposes. During the pandemic, it is best to clarify on what the two masks are meant for and when and where they should be used.

A surgical mask was not meant to protect you from harmful particles in the environment, as that is what respirators are meant to do. A surgical mask's purpose was to protect others from you. A 2013 study published in the journal Plos Pathogens [D.K Milton, M.P. Fabian, B.J. Cowling, M.L. Granthm, J.J. McDevitt (2013)] showed that scientists collected examples of exhaled particles from 38 influenza virus patients. The number of particles that passed 
through with and without a mask are shown in Table 1. As a result of this experiment, wearing a surgical mask can significantly decrease the risk of spreading your own disease and viruses to others.

Table 1. Particles that escaped into the air with and without a mask

\begin{tabular}{|c|c|c|c|c|c|}
\hline Percentiles: & Min & $25^{\text {th }}$ & Median & $75^{\text {th }}$ & Max \\
\hline Age & 18 & 18 & 19 & 20 & 54 \\
\hline Days since onset & 0 & 1 & 2 & 3 & 5 \\
\hline Nasopharyngeal swab copy number & $1.7 * 10^{3}$ & $8.3 * 10^{4}$ & $4.2 * 10^{5}$ & $1.8^{*} 10^{6}$ & $3.4 * 10^{7}$ \\
\hline Coarse particle copy number with mask & 0 & 0 & 0 & 0 & $7.7 * 10^{1}$ \\
\hline Coarse particle copy number with no & 0 & 0 & 0 & $3.7 * 10^{1}$ & $2.9 * 10$ \\
\hline mask & & & & & \\
\hline Fine particle copy number with mask & 0 & 5 & $2.2 * 10^{1}$ & $2.5^{*} 10^{2}$ & $2.4 * 10^{4}$ \\
\hline Fine particle copy number no mask & 0 & $1.1 * 10^{1}$ & $1.1 * 10^{2}$ & $5.6 * 10^{2}$ & $1.3 * 10^{5}$ \\
\hline
\end{tabular}

Taking the median as an example, although no particles escaped into the air with coarse particle copy numbers, there is a big difference between fine particle copy numbers with masks and without masks. With a mask, the number of particles that escaped was 5 times less than the particles that escaped without a mask.

Comparing surgical masks to N95s, a surgical mask is more for protecting others, while N95s can do both. However, N95 masks are more expensive, and more in demand than surgical masks as N95s are most needed by the frontline healthcare workers, which was why surgical masks are more recommended to the public. Before COVID19 , surgical masks were mainly used for protecting the patient from any risk of your own particles during a surgery, which is why it has low protection against particles trying to enter the mask. They are also loose fit, so in a contaminated environment, particles can easily enter the mask through the gaps on the side of the mask, but particles trying to exit the mask will be strictly blocked.

Overall, N95 masks work better and is more effective, but due to its high demand surgical masks can be used as an alternative.

\section{How to decontaminate N95s and recharge them for second use}

N95 masks are currently in high demand due to the COVID-19 global pandemic. They are needed by healthcare workers and everyday citizens to protect themselves and others from COVID. of the polypropylene in the mask will degrade, causing the filtering efficiency of the entire mask to decrease. N95s are meant to be disposable because they may have been contaminated by COVID, or more commonly, the efficiency of the mask has already degraded but due to the masks being in high demand, many scientists are looking to reuse and decontaminate the masks. Over the course of being used, the charge of the polypropylene in the mask will degrade, causing the filtering efficiency of the entire mask to decrease. 
Many articles and videos show how to decontaminate the mask with alcohol, UV radiation, ozone, and many more, but once the mask has been decontaminated, they lose any remaining charge left on the polypropylene, causing the mask to be nothing more than fabric.

However, there is a way to decontaminate and recharge the masks, which will be shown in this experiment. A plastic ball was used to serve as a head [E. Hossain, S. Bhadra, H. Jain, S. Das, A. Bhattacharya, S. Ghosh, D. Levine (2020)]. The mask was then strapped on the ball and air was sucked through the mask with a vacuum that produced a maximum flow of $30 \mathrm{~L} / \mathrm{min}$, although for most experiments the air was controlled at $10 \mathrm{~L} / \mathrm{min}$ to mimic an average person's breathing. The particles that passed through the mask was then counted by a particles counter, as shown in Figure 1(a).

(a)

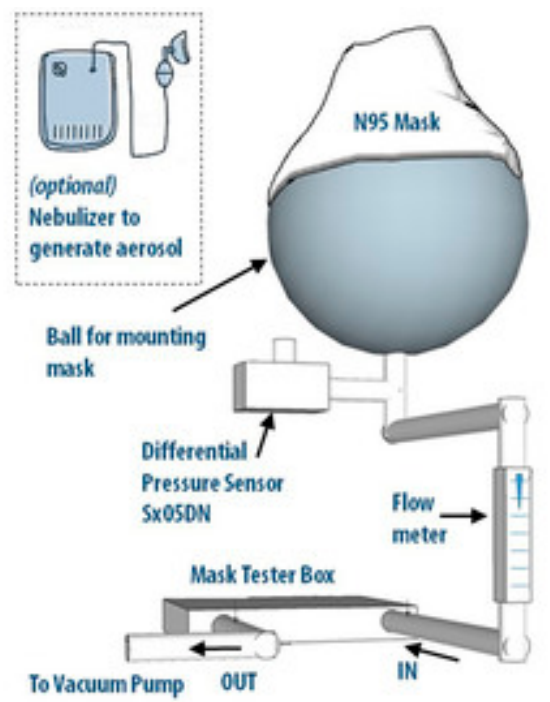

(b)

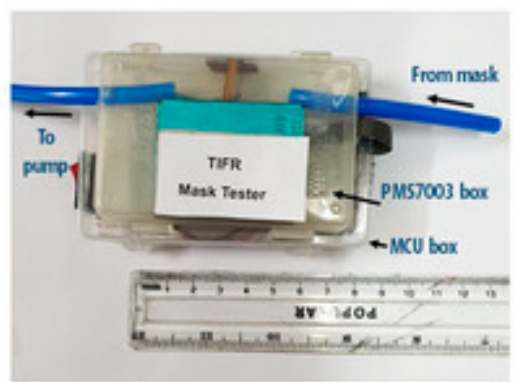

(c)

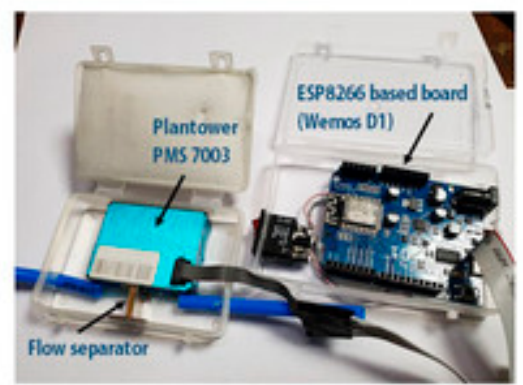

Figure 1: Diagram of the particle measuring process.

The charge of the mask was then measured with an electrometer by placing the mask in a metal cup that was isolated from the ground by an insulator surface. They were recharged by putting them between two metal plate electrodes, which were connected to the high and low output terminals in a power supply. The low input was grounded and a voltage of positive or negative polarity was applied from the high output of the source meter.

$$
\text { [a] }
$$
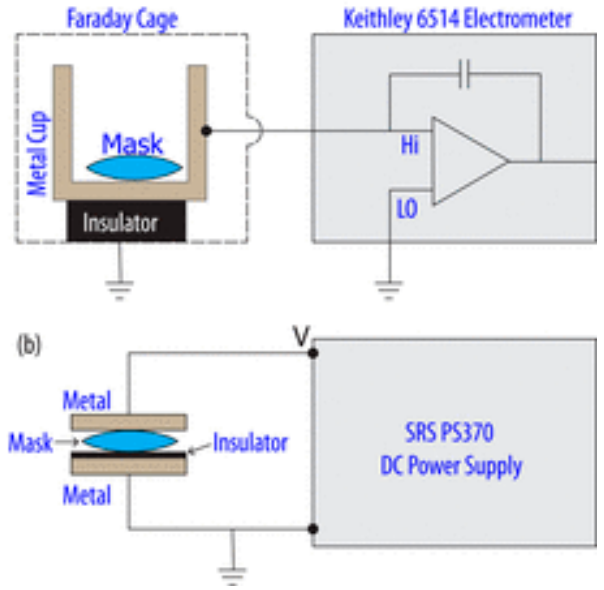

Figure 2: The process of measuring the charge and recharging the mask. 
To measure the efficiency of the masks after they were recharged, an unused KN95 mask with an efficiency of $95 \%$ $\pm 1 \%$ was used to test the efficiency before and after the mask was recharged. After testing it is efficiency, the mask was washed at 40 degrees Celsius in a conventional washing machine with detergent. Such methods would be expected to remove the layer of SARS-Cov-2 virus, which causes COVID-19. After the mask dried, its efficiency was measured to be $75 \% \pm 1 \%$. The method was then recharged for 60 minutes using the method shown in Figure 2(b), with a voltage of $2000 \mathrm{~V}$. Table 2 shows the collective effectiveness of recharging the masks with different sanitization methods.

Table 2: Filtration efficiency before recharging and after recharging with different sanitization methods.

\begin{tabular}{|c|c|c|}
\hline Sanitization Method & $\begin{array}{c}\text { Filtration efficiency (number of } \\
\text { particles blocked divided by total } \\
\text { particles) }\end{array}$ & \\
\hline (mask brand) & Before Recharge (\%) & After Recharge $(\%)$ \\
\hline Ethanol KN95 & 90 plus minus 1 & 96 plus minus 1 \\
\hline Boiling water KN95 & 74 plus minus 1 & 86 plus minus 1 \\
\hline Washing machine KN95 & 75 plus minus 1 & 95 plus minus 1 \\
\hline Steam exposed & 77 plus minus 1 & 86 plus minus 1 \\
\hline (Venus V-4420N95) & 77 plus minus 1 & 86 plus minus 1 \\
\hline Ethanol (Magnum N95) & 50 plus minus 1 & 86 plus minus 1 \\
\hline
\end{tabular}

Since the loss of charge from the N95 masks are known to impact the efficiency of the N95, the possibility of reusing the N95 masks was investigated. The results from this experiment shows that it was possible to recharge a mask and regain its efficiency even after the mask has lost its charge. However, it was unknown as to how many times the recharging process can be repeated, and whether the efficiency of the mask will decrease over the number of times the mask is recharged. Nonetheless, being able to recharge the mask at least once will have a significant impact on hospitals where mask supplies are insufficient.

\section{Further Studies and Applications}

The studies of these experiments will help benefit everyone who is affected by COVID-19. The information of these experiments and observations can be used to help billions of people make the right choices between different masks, and how to use them efficiently. However, the results of these experiments and observations might be different from real life applications. Firstly, the experiment on the effectiveness of the surgical mask took on place in 2018, and was tested using the influenza virus, so COVID-19 might have different effects on the surgical masks. However, the properties of the surgical masks are still the same, and they are still designed to keep your own particles from escaping the mask. Secondly, the experiment on recharging and reusing N95 masks is mostly accurate, but they have only tested the recharging efficiency with a few decontamination methods. The use of different decontamination methods than shown in the table can result in difference of mask filtering efficiency. Also, the more times this experiment is repeated with the same or different sanitization methods, the more accurate the results will be, or if using different sanitization methods, the wider the range of sanitization types and its filtering efficiency.

\section{Conclusions}

In conclusion, the physics behind N95 masks is very different from what you might have imagined and from the designs of other masks. This is shown through the properties of Spunbond and Meltblown polypropylene, and recharging N95 masks after decontaminating them so they can be reused. This is a useful process because it will create 
an alternative to fulfill the high demand for N95 masks. Being able to understand the physics behind N95 masks will help decrease the risk of COVID-19 for everyone.

\section{Acknowledgements}

I acknowledge my friend Dima for keeping me entertained while I was writing this paper.

\section{References}

[1] 2020 | T.H.I. U.S. Embassy Tbilisi | 12 August, U.S. Embassy in Georgia (2020).

[2] C. Clase, C.D. Lannoy, and S. Laengert, Brighter World (2020).

[3] D.K. Milton, M.P. Fabian, B.J. Cowling, M.L. Grantham, and J.J. McDevitt, PLOS Pathogens (2013).

[4] E. Hossain, S. Bhadra, H. Jain, S. Das, A. Bhattacharya, S. Ghosh, and D. Levine, AIP Publishing (2020). 